\title{
An estimate of average lower mantle conductivity by wavelet analysis of geomagnetic jerks
}

\author{
Mioara Mandea Alexandrescu, ${ }^{1}$ Dominique Gibert, ${ }^{2}$ Jean-Louis Le Mouël, ${ }^{1}$ \\ Gauthier Hulot, ${ }^{1}$ and Ginette Saracco ${ }^{2}$
}

\begin{abstract}
It has recently been proposed that geomagnetic jerks observed at the Earth's surface could be viewed as singularities in the time behavior of the geomagnetic field with a regularity of about 1.5 when wavelet analyzed. Such a signal should have suffered some distortion when diffusing from the core-mantle boundary (CMB) through the conducting mantle. Assuming that the upper mantle is an insulator and given the electromagnetic time constant of the mantle, we compute the distortion that a pure singularity introduced at the CMB suffers as it traverses the mantle. We compute this distortion through its effects on the so-called ridge functions extracted from the wavelet transform of the signal. This distortion is very similar to the small but significant one that we observe in real data. We therefore speculate that jerks must have been pure singularities at the base of the mantle and infer an average estimate for the mantle electromagnetic time constant from the way the signal is distorted by fitting the synthetic ridge functions to the experimental ones. Assuming, for example, a thickness of $2000 \mathrm{~km}$ for a uniform lower conducting mantle, we find an electrical conductivity smaller than $10 \mathrm{~S} \mathrm{~m}^{-1}$. This value is in reasonable agreement with values derived from high-pressure experiments for a silicate mantle.
\end{abstract}

\section{Introduction}

Variations of the geomagnetic field are the sum of external variations (whose sources are located in the ionosphere or farther out in the magnetosphere) and the so-called secular variation of the main field of internal origin. The secular variation gives information on the motions in the fluid core [e.g., Hulot et al., 1992] and provides tests for the different models of field generation which have been put forward. Also, and this is the subject of the present paper, the shortest-period components of the secular variation reaching the Earth's surface which are not completely screened by the mantle make it possible to estimate some average value of its electrical conductivity.

Examination of geomagnetic data from worldwide observatories has revealed sudden changes of the trend of the secular variation, which have been called "geomagnetic jerks" or "secular variation impulses" and have been discussed by a number of authors [Courtillot et al., 1978; Malin and Hodder, 1982; Malin et al., 1983;

\footnotetext{
${ }^{1}$ Institut de Physique du Globe de Paris, France.

${ }^{2}$ Géosciences Rennes - CNRS/INSU, Université de Rennes 1, France.
}

\section{Copyright 1999 by the American Geophysical Union.}

Paper number 1999JB900135.

0148-0227/99/1999JB900135\$09.00
Courtillot and Le Mouël, 1984; Kerridge and Barraclough, 1985; Gavoret et al., 1986; Gubbins and Tomlinson, 1986; Whaler, 1987; Golovkov et al., 1989; McLeod, 1992; Stewart and Whaler, 1992]. These former analyses have shown the global character and the internal origin of these events. In order to recover more accurate information about these events (time of occurrence, duration, distribution, and other characteristics), we recently applied a wavelet analysis to geomagnetic time series from $\sim 100$ observatories [Alexandrescu et al., $1995,1996]$. We found that seven, and only seven, such events took place during the 1900-1990 period, two of which (1969 and 1978) at least could be described as global in character. The events reveal a singular behavior with a regularity (see equation (27)) close to 1.5. A more recent geomagnetic jerk occuring in 1991 has been discussed by Macmillan [1996] and De Michelis et al. [1998] and also has a worldwide character [Le Huy et al., 1998].

The identification of these geomagnetic jerks originating within the core, observed at the top of the mantle, and having a short time constant produced a flurry of interest in the topic of lower mantle conductivity. Ducruix et al. [1980], Achache et al. [1980, 1981], Backus [1983], Courtillot et al. [1984], and McLeod [1994] took this observation as a stimulus to reconsider mantle conductivity estimates by the theory of electromagnetic diffusion through the mantle [Runcorn, 1955]. Ducruix et al. [1980] and Achache et al. [1980, 1981] 
used the secular variation impulse to constrain deep mantle conductivity "from the bottom" and the 11-yr response to the external field to constrain conductivity "from the top." They proposed that the conductivity is everywhere smaller than a few hundred $\mathrm{S} \mathrm{m}^{-1}$. Backus [1983] developed and applied a mantle filter theory, concluding that an arbitrarily large value of the conductivity is still allowed in the deepest layers of the mantle. Courtillot et al. [1984], using the formalism of Backus [1983] in relation to the 1969 geomagnetic jerk, concluded that the electrical conductivity is probably lower than a few hundred $\mathrm{S} \mathrm{m}^{-1}$ in $>97 \%$ of the mantle volume. Finally, McLeod [1994] derived a global geomagnetic response function consistent with a conductivity of about $3 \mathrm{~S} \mathrm{~m}^{-1}$ at the core-mantle boundary (CMB).

\section{Diffusion Through the Conductive Mantle}

In this section our work is complementary to that of previous investigators [Smylie, 1965; Backus, 1983; Benton and Whaler, 1983] who also examined the case of the diffusion of a rapid impulsive change produced at the CMB. In the following, a similar approach is used with the view of computing the response of the mantle to a jerk signal (see section 5) with a noninteger regularity. The two-layer mantle model is spherically symmetric and consists of an internal shell $\left(r_{c}<r \leq r_{\sigma}\right)$ of conductivity $\sigma$ and an insulating outer shell $\left(r_{\sigma}<r \leq\right.$ $R_{E}$ ) where $r_{c}$ and $R_{E}$ are the radius of the outer core and of the Earth, respectively. For the sake of simplicity, we consider the conductivity of the lower shell uniform; in fact, what we are interested in is the electromagnetic time constant $\approx \mu \sigma\left(r_{\sigma}-r_{c}\right)^{2}$ of the mantle, where $\mu$ is the magnetic permeability of free space.

The problem we address is to obtain the magnetic field at the Earth's surface from the time-varying magnetic field given at the CMB. The poloidal component (which is the one of interest in the present problem) of the magnetic field $\mathbf{B}(\mathbf{r}, t)$ in the mantle may be studied independently of the toroidal component and can be derived from a scalar potential $p(\mathbf{r}, t)$ [e.g., Le Mouël, 1976; Backus et al., 1996]:

$$
\mathbf{B}(\mathbf{r}, t)=\nabla \times[\nabla \times \mathbf{r} p(\mathbf{r}, t)],
$$

where $\mathbf{r}$ is the radius vector with spherical coordinates $(r, \theta, \phi)$. The potential $p$ satisfies a diffusion equation in the internal conducting shell:

$$
\nabla^{2} p=\mu \sigma \frac{\partial p}{\partial t} \quad\left(r_{c}<r \leq r_{\sigma}\right)
$$

and the Laplace equation in the insulating upper part of the mantle:

$$
\nabla^{2} p=0 \quad\left(r_{\sigma}<r \leq R_{E}\right) .
$$

In both shells the potential may be written as a series of spherical harmonics $Y_{n}^{m}(\theta, \varphi)$,

$$
p(\mathbf{r}, t)=\sum_{n} \sum_{m} s_{n}^{m}(r, t) Y_{n}^{m}(\theta, \varphi),
$$

where $s_{n}^{m}$ coefficients must satisfy partial differential equations derived from (2) and (3). In the conducting shell $\left(r_{c}<r \leq r_{\sigma}\right)$ we have

$$
\frac{1}{r} \frac{\partial^{2}}{\partial r^{2}}\left(r s_{n}^{m}\right)-\frac{n(n+1)}{r^{2}} s_{n}^{m}=\mu \sigma \frac{\partial s_{n}^{m}}{\partial t}
$$

and in the insulating shell $\left(r_{\sigma}<r \leq R_{E}\right)$,

$$
\frac{1}{r} \frac{\partial^{2}}{\partial r^{2}}\left(r s_{n}^{m}\right)-\frac{n(n+1)}{r^{2}} s_{n}^{m}=0 .
$$

Assuming a field of internal origin whose sources are located inside the sphere $r=r_{\sigma},(6)$ gives

$$
s_{n}^{m}\left(R_{E}, t\right)=\left(\frac{r_{\sigma}}{R_{E}}\right)^{n+1} s_{n}^{m}\left(r_{\sigma}, t\right) .
$$

The link between $s_{n}^{m}\left(r_{c}, t\right)$ and $s_{n}^{m}\left(r_{\sigma}, t\right)$ is more complicated to derive due to the presence of the time variable in the diffusion equation (5). It can be shown [Smylie, 1965] that the relationship sought is a convolution integral:

$$
s_{n}^{m}\left(r_{\sigma}, t\right)=\frac{r_{c}}{r_{\sigma}} \int_{0}^{\infty} s_{n}^{m}\left(r_{c}, \xi\right) k_{n}(t-\xi) \mathrm{d} \xi
$$

where the causal convolution kernels $k_{n}(t)$ have the following form:

$$
k_{n}(t)=\sum_{i} \kappa_{n, i} \exp \left(-\frac{t}{\tau_{n, i}}\right) .
$$

The coefficients $\kappa_{n, i}$ and the time constants $\tau_{n, i}$ are such that

$$
M_{n}\left(r_{c}, \tau_{n, 2}^{-1}\right)=0
$$

and

$$
\kappa_{n, 2}=\frac{r_{\mathrm{c}}}{r_{\sigma}}\left[\frac{\mathrm{d} M_{n}\left(r_{c}, q\right)}{\mathrm{d} q}\right]_{q}^{-1},
$$

where $q=\tau_{n, i}^{-1}$; the function $M_{n}(r, q)$ is, within a multiplicative factor, the Laplace transform of $s_{n}^{m}$ and satisfies the differential equation

$$
\frac{\mathrm{d}^{2} M_{n}}{\mathrm{~d} r^{2}}-\frac{n(n+1)}{r^{2}} M_{n}(r, q)=\mu \sigma q M_{n}(r, q)
$$

subject to the boundary conditions:

$$
\begin{aligned}
M_{n}\left(r_{\sigma}, q\right) & =1, \\
\left(\frac{\mathrm{d} M_{n}}{\mathrm{~d} r}\right)_{r=r_{\sigma}} & =-\frac{n}{r_{\sigma}} .
\end{aligned}
$$

We can find an analytical expression for the function $M_{n}(q, p)$ [Petıau, 1955; Smylie, 1965]: 


$$
\begin{aligned}
M_{n}(r, q)= & \frac{i \pi}{4} \gamma\left[r r _ { \sigma } \left(H_{n-\frac{1}{2}}^{(2)}\left(\gamma r_{\sigma}\right) H_{n+\frac{1}{2}}^{(1)}(\gamma r)-\right.\right. \\
& \left.\left.-H_{n-\frac{1}{2}}^{(1)}\left(\gamma r_{\sigma}\right) H_{n+\frac{1}{2}}^{(2)}(\gamma r)\right)\right]^{1 / 2}
\end{aligned}
$$

where $\gamma^{2}=-\mu \sigma q, H_{\beta}^{(1)}$ and $H_{\beta}^{(2)}$ are Hankel functions of the first and second kinds, respectively.

By combining (7) and (8), we obtain the relationship between the spherical harmonic coefficients of the potential at the CMB and at the surface of the Earth:

$$
\begin{aligned}
s_{n}^{m}\left(R_{E}, t\right)= & \left(\frac{r_{\sigma}}{R_{E}}\right)^{n+1} \frac{r_{c}}{r_{\sigma}} \int_{0}^{\infty} s_{n}^{m}\left(r_{c}, \xi\right) . \\
& \cdot k_{n}(t-\xi) \mathrm{d} \xi .
\end{aligned}
$$

We now derive expressions for the magnetic field measured at the Earth's surface and created by a magnetic jerk occurring at the CMB. From the results just derived (equations (4) and (11)) we have

$$
\begin{aligned}
& \mathbf{B}(\mathbf{r}, t)=\nabla \times[\nabla \times \mathbf{r} p(\mathbf{r}, t)] \\
\mathbf{B}(\mathbf{r}, t)= & \sum_{n} \sum_{m} \nabla \times\left[\nabla \times \mathbf{r}\left(\frac{r_{\sigma}}{r}\right)^{n+1} \frac{r_{c}}{r_{\sigma}} Y_{n}^{m}(\theta, \varphi)\right. \\
& \left.\int_{0}^{\infty} s_{n}^{m}\left(r_{c}, \xi\right) k_{n}(t-\xi) \mathrm{d} \xi\right]
\end{aligned}
$$

subject to the condition that the field is computed outside the conducting shell, i.e., $r>r_{\sigma}$.

We shall assume that the time variation of the coefficients $s_{n}^{m}$ at the CMB is independent of both degree $n$ and order $m$. This hypothesis is based on the observations that the regularity of the signals recorded at the observatories is the same all over the Earth within the uncertainty estimates. We can write

$$
s_{n}^{m}\left(r_{c}, t\right)=g_{n}^{m}\left(r_{c}\right) j(t),
$$

where the $g_{n}^{m}\left(r_{c}\right)$ coefficients define the geometry of the field emitted at the CMB and the function $j(t)$ describes the time behavior (i.e., the jerk in sections 3 and 5) of this field.

Thus the convolution product may be removed from the summation over $m$, and (18) becomes

$$
\begin{aligned}
\mathbf{B}(\mathbf{r}, t)= & \sum_{n} \int_{0}^{\infty} j(\xi) k_{n}(t-\xi) \mathrm{d} \xi . \\
& \cdot\left\{\nabla \times\left[\nabla \times \mathbf{r}\left(\frac{r_{\sigma}}{r}\right)^{n+1} \frac{r_{c}}{r_{\sigma}} .\right.\right. \\
& \left.\left.\cdot \sum_{m} g_{n}^{m}\left(r_{c}\right) Y_{n}^{m}(\theta, \varphi)\right]\right\},
\end{aligned}
$$

again with the condition $r>r_{\sigma}$. Defining in $\left(r>r_{\sigma}\right)$, $p_{n}$ as

$$
p_{n}(\mathbf{r})=\left(\frac{r_{\sigma}}{r}\right)^{n+1} \frac{r_{c}}{r_{\sigma}} \sum_{m} g_{n}^{m}\left(r_{c}\right) Y_{n}^{m}(\theta, \varphi),
$$

and the poloidal field of degree $n, \mathbf{B}_{n}(\mathbf{r})$, by

$$
\mathbf{B}_{n}(\mathbf{r})=\nabla \times\left[\nabla \times \mathbf{r} p_{n}(\mathbf{r})\right],
$$

(20) may be rewritten as

$$
\mathbf{B}(\mathbf{r}, t)=\sum_{n} \mathbf{B}_{n}(\mathbf{r}) \int_{0}^{\infty} j(\xi) k_{n}(t-\xi) \mathrm{d} \xi .
$$

At the surface of the Earth, (23) reduces to

$$
\begin{aligned}
\mathbf{B}\left(R_{E}, \theta, \phi, t\right)= & \sum_{n} \mathbf{B}_{n}\left(R_{E}, \theta, \phi\right) . \\
& \cdot \int_{0}^{\infty} j(\xi) k_{n}(t-\xi) \mathrm{d} \xi .
\end{aligned}
$$

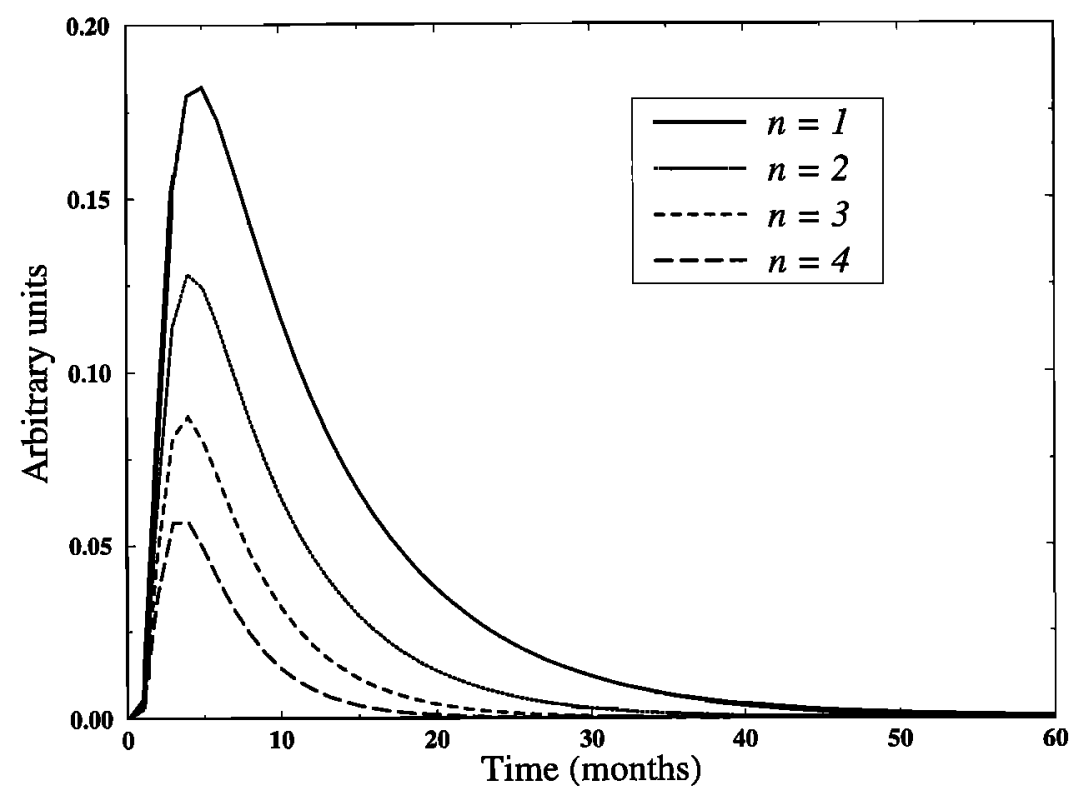

Figure 1. Kernels $k_{n}$ for $n=1,2,3,4, r_{\sigma}-r_{c}=2000 \mathrm{~km}$, and $\sigma=16 \mathrm{Sm}^{-1}$. 


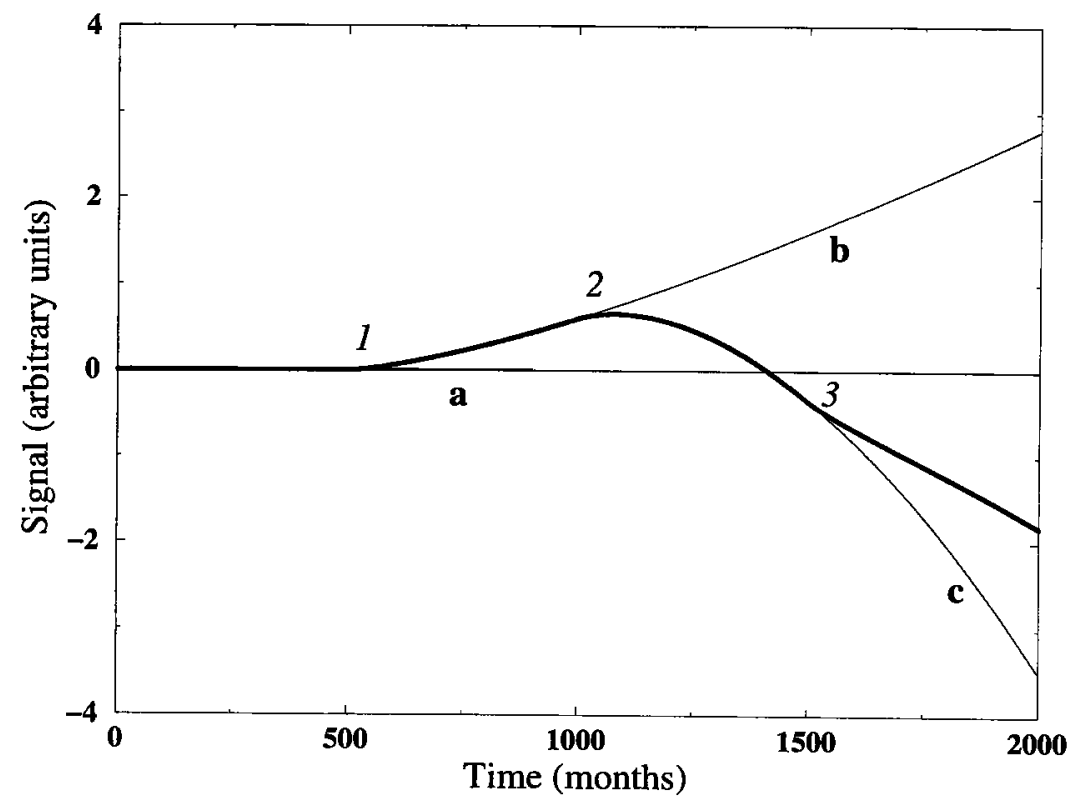

Figure 2. Thick solid line is synthetic signal composed of three jerks located at $t_{0}=$ $(500,1000,1500)$ with regularities $\alpha=(1.4,1.7,1.5)$ and amplitudes $\beta=(+1.0,-0.5,+1.5)$. The thin solid lines are extrapolations of the signal obtained by extinguishing several jerks: line $a$ is what the signal would have been in the absence of jerks 1,2 , and 3 ; line $\mathrm{b}$ is what the signal would have been if jerks 2 and 3 were absent, and line $c$ is what the signal would have been if jerk 3 was absent.

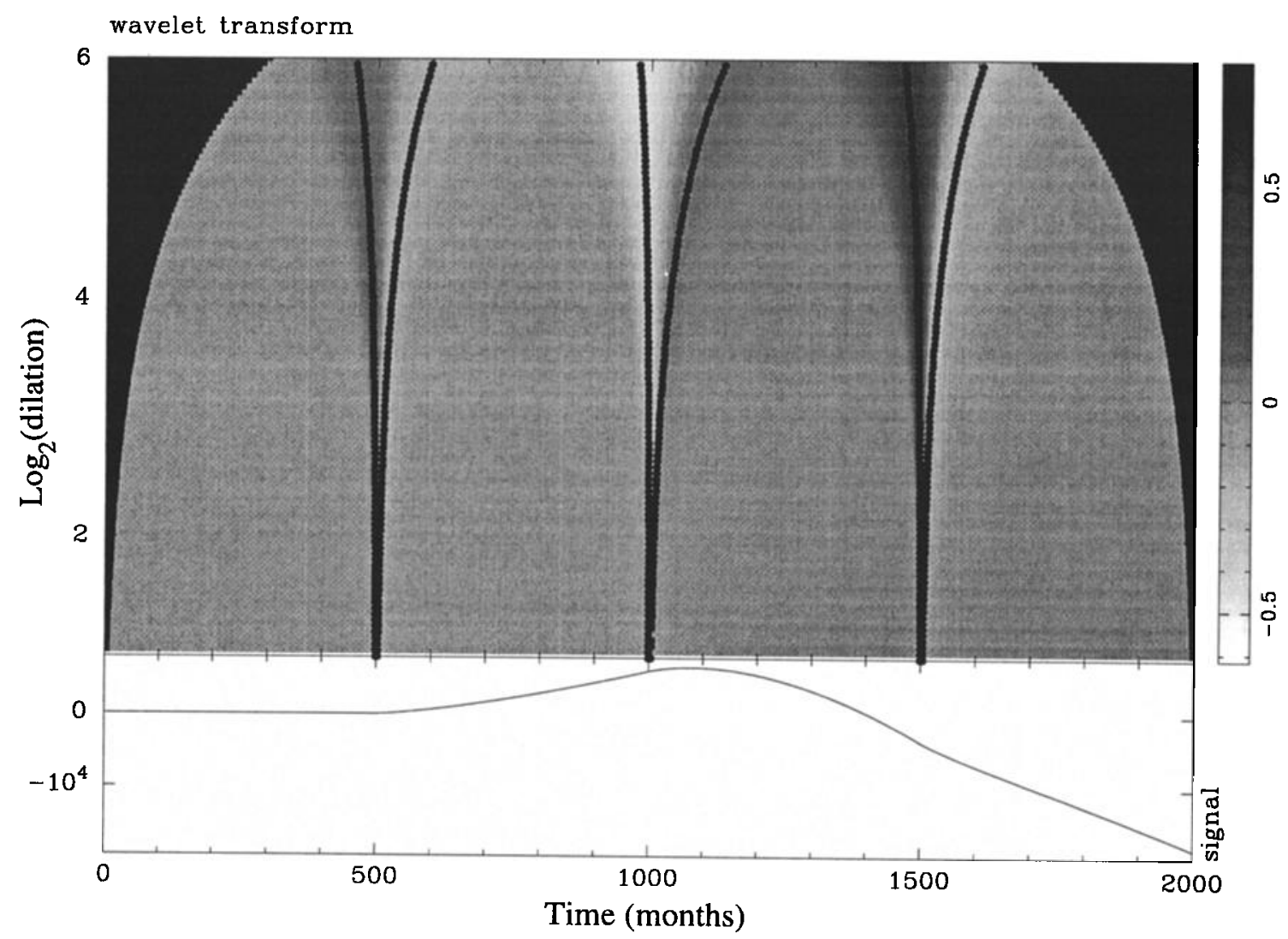

Figure 3. (top) Wavelet transform of the (bottom) signal shown in Figure 2. The analyzing wavelet is the third time derivative of a Gaussian (see Alexandrescu et al. [1995] for details). Observe the three cone-like patterns converging toward the starting dates of the three jerks present in the analyzed signal. The solid lines are lines of extrema of the wavelet transform from which the ridge functions shown in Figure 4 are extracted. 

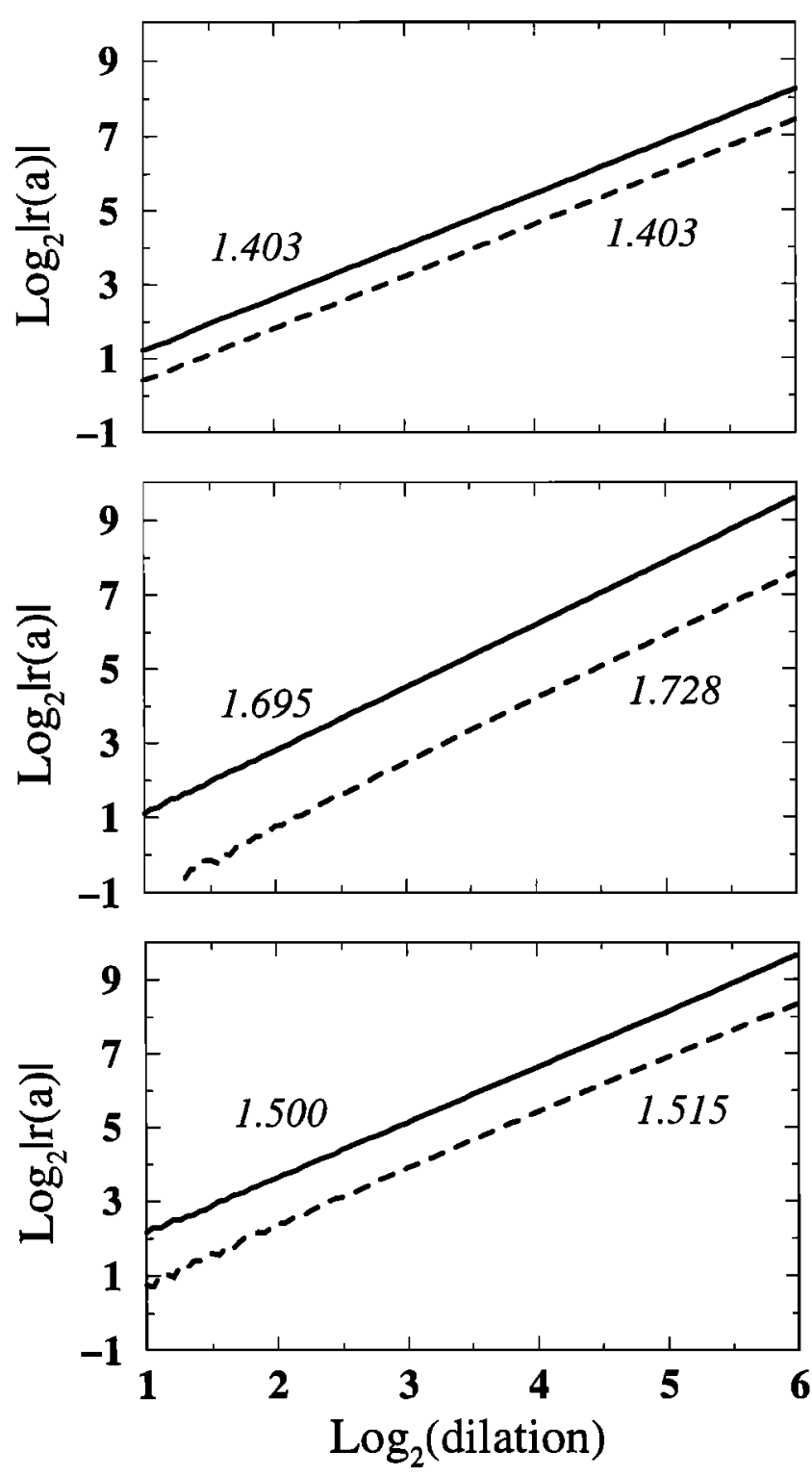

Figure 4. Ridge functions $r(a)$ extracted from the wavelet transform shown on Figure 3 and corresponding to the jerks labeled (top) 1 , (middle) 2 , and (bottom) 3 in Figure 2. The solid lines are the ridge functions located on the left part of the cone pattern of the wavelet transform, and the dashed lines are the ridge functions located on the right. The numbers are the slopes obtained by least squares fitting of a straight line. Observe that these slopes fall very near the theoretical values $(1.4,1.7$, and 1.5 respectively).

Note that $\mathbf{B}_{n}$ are also taken at the surface of the Earth and that (24) may be used to account for the geometry of a time-varying field measured on the Earth. In this way we incorporate the spatial structure of the 1969 jerk into our synthetic data. This is important in practice since, depending on the geographical position on the Earth, the relative amplitudes of the $\mathbf{B}_{n}$ can vary and the temporal variations of the field $\mathbf{B}$ may differ from one place to another, since the kernels $k_{n}$ display some dependence upon the degree $n$, as shown in Figure 1 .

\section{Ridge Functions}

The data used in this study are a set of ridge functions (see section 4), and what we actually have to produce for comparison are synthetic ridge functions. A ridge function is a subset of the continuous wavelet transform of a signal $f(t)$ which contains the information useful for characterizing abrupt variations in the signal [Grossmann, 1986]. The continuous wavelet transform of $f(t)$ is defined by the convolution product

$$
W f[t, a]=\int_{-\infty}^{+\infty} f(\xi) \psi_{a}(t-\xi) \mathrm{d} \xi,
$$

where the wavelets $\psi_{a}(t)$ are obtained by dilating an analyzing wavelet $\psi(t)$,

$$
\psi_{a}(t)=\frac{1}{a} \psi\left(\frac{t}{a}\right)
$$

with a dilation $a>0$. In the present study the analyzing wavelet is the third time derivative of a Gaussian as already used by Alexandrescu et al. [1995, 1996]. Among the numerous attractive properties of the wavelet transform (see, for instance, Holschnerder [1995]), we exploit the fact that the wavelet transform acts like a mathematical zoom lens useful for analyzing the local homogeneity characteristics (i.e., the local self-similar behavior) of a signal.

In the present study we shall focus on so-called jerk signals whose general expression is

$$
j\left(t \mid \alpha, \beta, t_{0}\right)=\beta \mathrm{H}\left(t-t_{0}\right)\left(t-t_{0}\right)^{\alpha},
$$

where $H(t)$ is the Heaviside distribution, $\beta$ is an amplitude factor, $t_{0}$ is the starting date of the jerk, and $\alpha$ is its regularity. The larger the regularity, the smoother the variation of the function. Let us, for instance, consider a signal $f(t)$ composed of three jerks:

$$
\begin{aligned}
f(t)= & j(t \mid 1.4,1,500)+j(t \mid 1.7,-0.5,1000)+ \\
& +j(t \mid 1.5,1.5,1500) .
\end{aligned}
$$

This signal is shown on Figure 2, and its wavelet transform is displayed on Figure 3. We observe that the wavelet transform has three cone-like features pointing (zooming) toward the starting dates $t_{0}$ of the three jerks composing the analyzed signal. It can be shown [Alexandrescu et al., 1995; Holschneıder, 1995] that each cone-like pattern possesses self-similar properties controlled by the homogeneity of its related jerk and that useful information can be recovered by simply taking the absolute value of the wavelet transform along only one of the lines of extrema of the cone-like pattern (Figure 3). The set of absolute values of the wavelet transform on a given line of extrema and ranked with respect to the dilation $a$ defines what will be referred to as a ridge function $r(\alpha)$. When plotted on a log-log diagram, the ridge functions are straight lines whose 


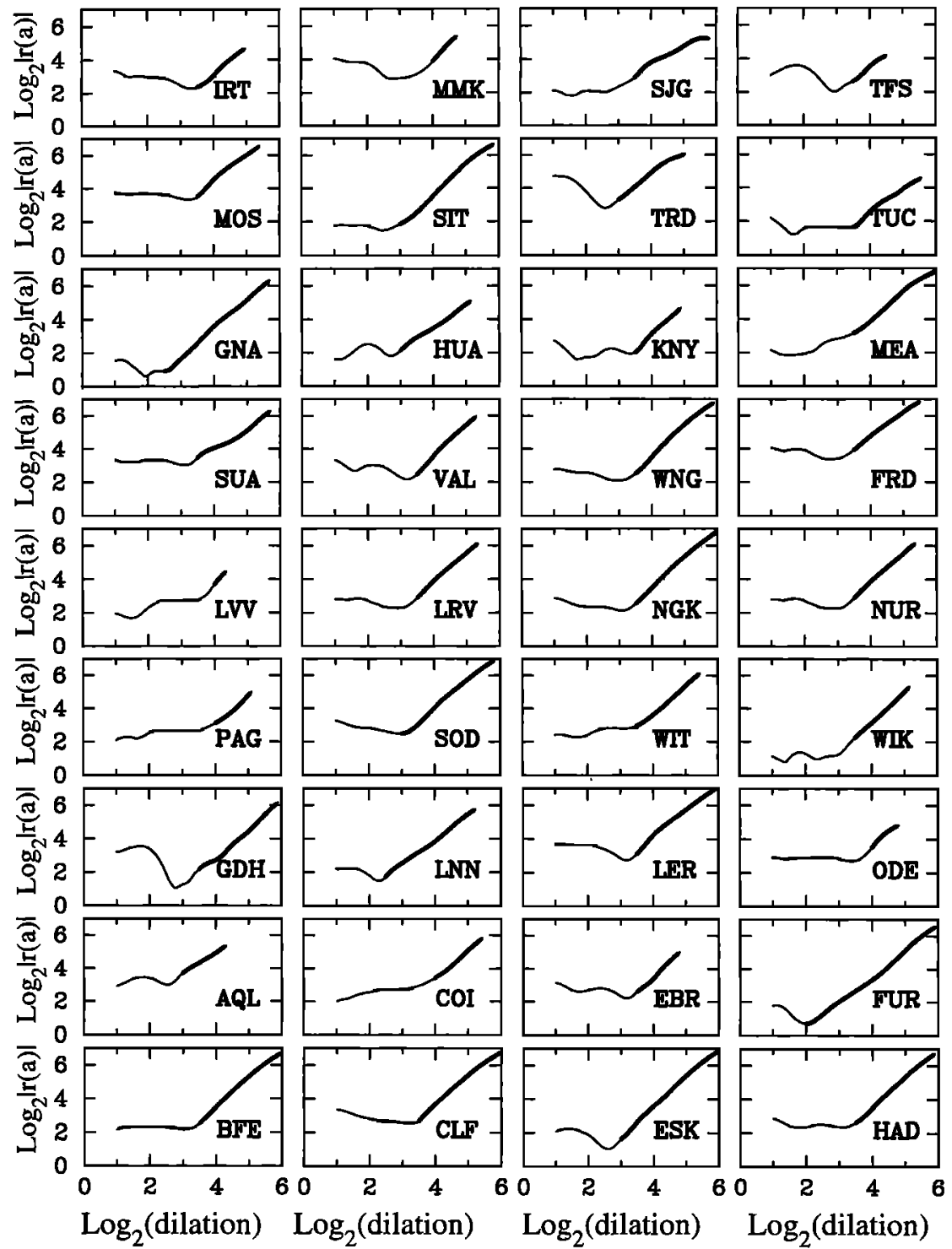

Figure 5. Thin lines are observed ridge functions for the 36 observatories considered in this study. Solid lines are parts of the ridge functions used in the inversion.

slopes equal the regularity $\alpha$ of the corresponding jerk (Figure 4).

\section{Data}

The data set used in this study is composed of the ridge functions (Figure 5) corresponding to the 1969 jerk and obtained through the wavelet analysis of the monthly mean series of 36 observatories [Alexandrescu et al., 1995, 1996]. We focus on the 1969 event because it is the best documented and appears to be less affected by fields of external origin than the others [see Alexandrescu et al., 1995, 1996]

The geographical coordinates of the observatories and their International Association of Geomagnetism and Aeronomy (IAGA) codes are listed in Table 1, and their global distribution is shown in Figure 6. For each observatory the ridge function was extracted from the wavelet transform computed for a linear combination of the $X$ (north) and $Y$ (east) components of the field, and the regularity $\alpha$ was found to vary between 1.5 and 1.7. The uncertainty associated with estimating the regularity in this way is about $10 \%$ [Alexandrescu et al., 1996]. The analyzed signal for the $l$ th observatory is

$$
H_{l}(t)=\mathbf{q}_{l} \bullet \mathbf{B}_{l}(t),
$$

where $\mathbf{B}_{l}(t)$ is the magnetic field at the $l$ th observatory and $q_{l}$ is a unit horizontal vector pointing in the direction of the jerk vector, estimated with an error of $\sim 5^{\circ}$ [see Alexandrescu et al., 1996]; the direction of this vector is given in Table 1 for each observatory.

\section{Synthetic Jerks}

Alexandrescu et al. [1995, 1996] supposed that the jerks observed at the Earth's surface were pure singu- 
Table 1. Observatories Considered in the Present Study

\begin{tabular}{llrrr}
\hline Code $^{a}$ & \multicolumn{1}{c}{ Name } & $\lambda^{b}$ & \multicolumn{1}{c}{$\varphi^{c}$} & $\varphi^{d}{ }^{d}$ \\
\hline AQU & L'Aquila & 42.383 & & \\
BFE & Brorfelde & 55.625 & 13.317 & 80 \\
CLF & Chambon la Forêt & 48.023 & 2.672 & 80 \\
COI & Coimbra & 40.222 & 351.583 & 90 \\
EBR & Ebro & 40.820 & 0.493 & 100 \\
ESK & Eskdalemuir & 55.317 & 356.800 & 80 \\
FRD & Fredericksurg & 38.205 & 282.627 & 50 \\
FUR & Furstenfeldbrück & 48.165 & 11.277 & 80 \\
GDH & Godhann & 69.252 & 306.467 & 90 \\
GNA & Gnangara & -31.783 & 115.950 & 80 \\
HAD & Hartland & 50.995 & 355.517 & 90 \\
HUA & Huancayo & -12.045 & 284.670 & 80 \\
IRK & Patrony & 52.167 & 104.450 & 60 \\
KNY & Kanoya & 31.420 & 130.882 & 110 \\
LER & Lerwick & 60.133 & 358.817 & 100 \\
LNN & Voyeykovo & 59.950 & 30.705 & 80 \\
LRV & Leirvogur & 64.183 & 338.300 & 90 \\
LVV & Lvov & 49.900 & 23.750 & 90 \\
MEA & Meanook & 54.617 & 246.667 & 110 \\
MMK & Loparskoye & 68.250 & 33.083 & 100 \\
MOS & Krasnaya Pakhra & 55.467 & 37.312 & 110 \\
NGK & Niemegk & 52.072 & 12.675 & 90 \\
NUR & Nurmijärvi & 60.508 & 24.655 & 90 \\
ODE & Stepanovka & 46.783 & 30.883 & 100 \\
PAG & Panagyuriste & 42.512 & 24.177 & 80 \\
SIT & Sitka & 57.058 & 224.675 & 110 \\
SJG & San Juan & 18.113 & 293.85 & 90 \\
SOD & Sodankylä & 67.368 & 26.6300 & 90 \\
SUA & Surlari & 44.680 & 26.253 & 90 \\
TFS & Dusheti & 42.092 & 44.705 & 70 \\
TRD & Trivandrum & 8.483 & 76.950 & 160 \\
TUC & Tucson & 32.247 & 249.167 & 80 \\
VAL & Valentia & 51.933 & 349.750 & 100 \\
WIK & Wien Kobenzl & 48.265 & 16.318 & 80 \\
WIT & Witteveen & 52.813 & 6.668 & 90 \\
WNG & Wingst & 53.743 & 9.073 & 90 \\
\hline & & & &
\end{tabular}

${ }^{a}$ According to the IAGA convention.

${ }^{b}$ Latitude of the observatory, in degrees.

${ }^{c}$ Longitude of the observatory, in degrees, positive eastward.

${ }^{d}$ Jerk direction, in degrees, positive eastward.

larities of the form given by (27) with straight ridge functions. Actually, when looking at experimental ridge functions (Figure 5), one observes a small downward curvature for many observatories. Such a curvature could be due to diffusion of the signal through the conductive mantle. We therefore speculate that the signal $j(t)$ emitted at the CMB is a pure jerk as given by (27) and that the curvatures observed in the ridge functions of the surface data are caused by diffusion modeled by the convolution equation (24). The transfer functions $k_{n}(t)$ are causal positive functions (Figure 1) and act as low-pass filters whose cutoff frequency is controlled by the electromagnetic time constant $\approx \mu \sigma\left(r_{\sigma}-r_{c}\right)^{2}$ and by the degree $n$ of the elementary harmonic considered. The larger the electromagnetic time constant, the stronger the filtering, and the ridge functions obtained through a wavelet analysis will depart more from a straight line when plotted in a log-log diagram. An example of this situation (computed for the Chambon la Forèt observatory coordinates, see below) is shown in Figure 7 for $r_{\sigma}-r_{c}=2000 \mathrm{~km}, \alpha=1.5$, and $\sigma=1,4,16,64 \mathrm{~S} \mathrm{~m}^{-1}$. We observe that the ridge functions present a downward curvature that increases with increasing $\sigma$. An intuitive assessment of this feature can be made by observing that the transfer function is a localized filter. Then, for sufficiently large dilations of the wavelet transform the filter is well approximated by a Dirac distribution, and the ridge function is asymptotically a straight line whose slope is the exponent $\alpha$ of the jerk produced at the CMB. For smaller dilations involving shorter timescales the finite duration of the filter cannot be ignored, and the signal is both smoothed and damped. Accordingly, this smoothing results in a larger regularity at the timescales considered, and the corre- 


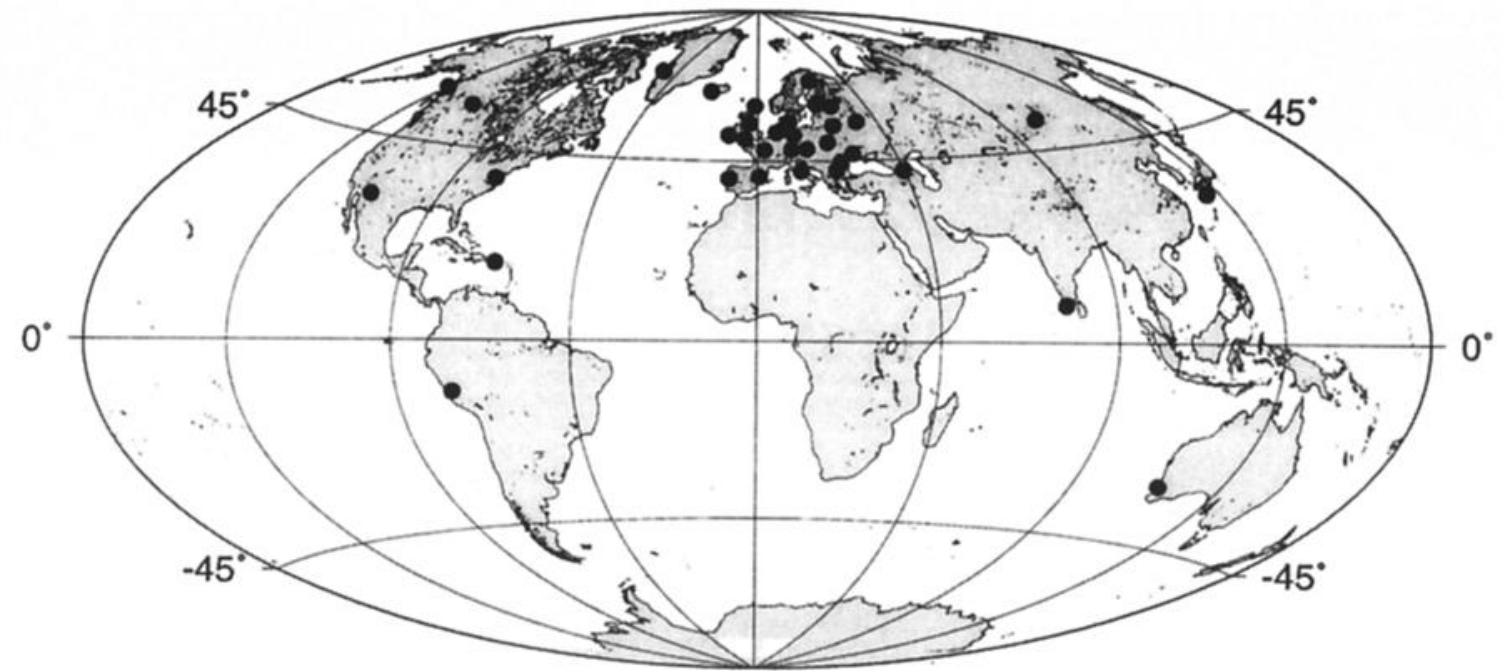

Figure 6. Distribution of the 36 observatories considered in the present study.

sponding part of the ridge function has a larger slope at the corresponding dilations. The ridge function of the surface jerk is therefore a curved line located below its asymptote which is the linear ridge function of the pure singularity jerk at the CMB.

In order to complete the expression of the synthetic jerk to be compared with the observed one, through their respective ridge functions, we need the harmonic field coefficients $\mathbf{B}_{n}$. We take as $\mathbf{B}_{n}$ the results of the analysis of Le Huy et al. [1998]. This study is basically a spherical harmonic expansion of the jump of the first time derivative of the field from the jump observed at the observatories. This is only a first approxima- tion for two reasons: (1) the analysis is performed from the data of the observatories, including the diffusion effect which we are trying to characterize; and (2) owing to the small number of observatories, the coefficients of the spherical harmonic expansion are not very accurate. Nevertheless, this expansion is acceptable for the needs of the present investigation (a two-step computation could be performed, i.e., recomputing the $\mathbf{B}_{n}$ after a first determination of the singularity at the CBM, but this appears to be unnecessary). The expansion of Le Huy et al. [1998] (limited to order and degree 4) compares well with previous studies [Malin and Hodder, 1982; Gubbins, 1984]. Moreover, at each observa-

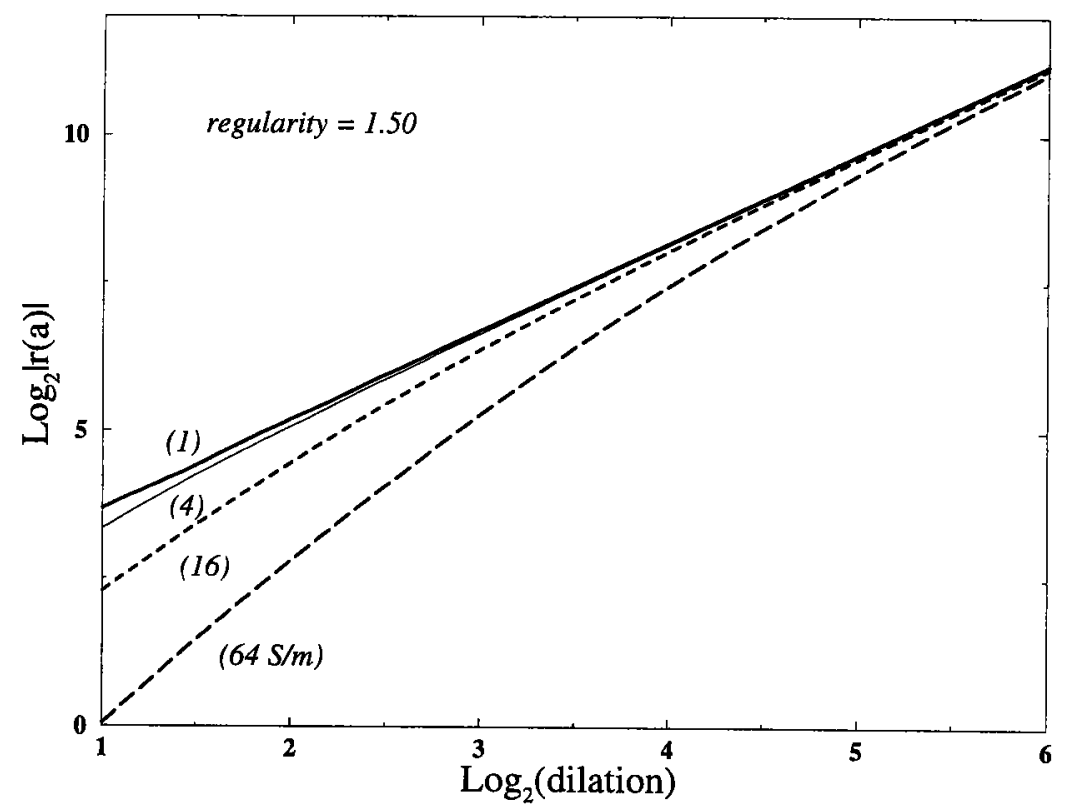

Figure 7. Ridge functions obtained for a surface jerk (corresponding to $\alpha=1.5$ at the CMB) filtered through a conducting shell with $r_{\sigma}-r_{c}=2000 \mathrm{~km}$ and for $\sigma=1,4,16$, and $64 \mathrm{Sm}^{-1}$ (see text for details). The curvature of the ridges is more pronounced for larger conductivities. 


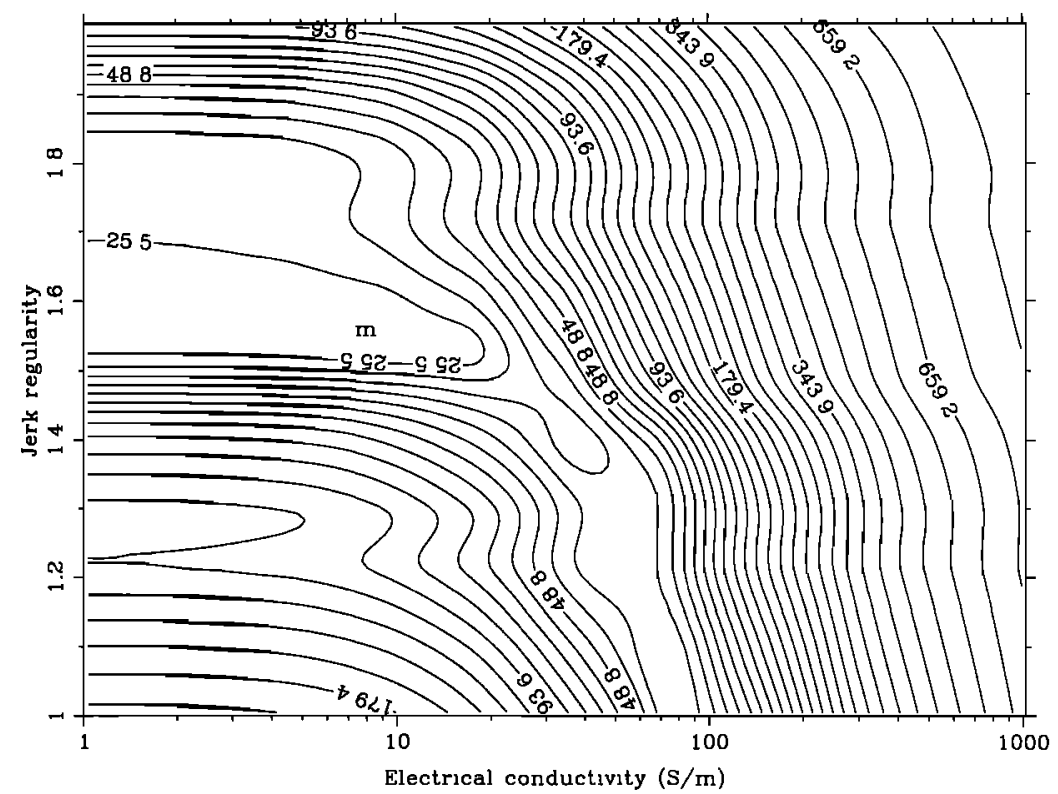

Figure 8. Misfit function $C(\alpha, \sigma)$ (equation (33)) obtained by fitting synthetic ridge functions to the experimental ridge functions of 36 observatories. The symbol $m$ is located at the absolute minimum (22.4) whose coordinates are $\alpha=1.55$, and $\sigma=8 \mathrm{Sm}^{-1}$.

tory the directions $\mathbf{q}_{l}$ of the jerk computed using (29) are close to those obtained by the wavelet analysis of the data. The synthetic ridge functions have been computed from synthetic series obtained from (24) subject to the same projection as in (29):

$$
\begin{aligned}
H_{l}\left(R_{E}, \theta_{l}, \phi_{l}, t\right)= & \sum_{n} \mathbf{q}_{l} \bullet \mathbf{B}_{n}\left(R_{E}, \theta_{l}, \phi_{l}\right) . \\
& \cdot \int_{0}^{\infty} j\left(\xi \mid \alpha, \beta, t_{0}\right) . \\
& \cdot k_{n}(t-\xi) \mathrm{d} \xi
\end{aligned}
$$

where $\left(\theta_{l}, \phi_{l}\right)$ are the coordinates of the $l$ th observatory and the directions of the unit vectors $\mathbf{q}_{l}$ are given in Table 1 (as noted, they are very close to those of $L e$ Huy et al. [1998]).

Equation (30) has been used to compute a collection of ridge functions $r_{l, \alpha, \sigma}(a)$ for the values $\left(\theta_{l}, \phi_{l}\right)$ of the coordinates of each of the 36 observatories and for $r_{\sigma}-$ $r_{c}=2000 \mathrm{~km}, \alpha \in[1.0,2.0]$ and $\sigma \in\left[1,1024 \mathrm{Sm}^{-1}\right]$. Then, for each $(\alpha, \sigma)$ pair belonging to these intervals, a misfit was computed:

$$
C(\alpha, \sigma)=\sum_{l} \sum_{j}\left[r_{l}\left(a_{j}\right)-r_{l, \alpha, \sigma}\left(a_{j}\right)\right]^{2},
$$

where the index $j$ runs over the discrete dilations for which the ridge function of a given observatory is available. A contour map of the misfit function $C(\alpha, \sigma)$ is shown in Figure 8. The absolute minimum (22.4) is located at the point $\alpha=1.55$ and $\sigma=8 \mathrm{~S} \mathrm{~m}^{-1}$. One can observe that the isovalues of $C(\alpha, \sigma)$ define a well-marked valley of minima surrounded by steep gradients, especially toward high conductivities. The valley widens toward small conductivities with a rather flat bottom indicating that the domain of acceptable solutions extends itself on the left of the absolute minimum. Values of $\alpha$ along the valley axis get smaller as $\sigma$ increases, leading to an increasingly more singular source: for $\alpha=1$ the first time derivative is discontinuous at $t_{0}$, implying an unrealistic discontinuity in the velocity flow at the CMB responsible for the jerk [Le Huy et al., 1998]. If we choose a regularity as high as possible, the conductivity $\sigma$ must be taken smaller than $10 \mathrm{~S} \mathrm{~m}^{-1}$. In any case, it is safe to say that on the basis of the misfit criterion we have adopted, the conductivity $\sigma$ is less than a few tens of $\mathrm{Sm}^{-1}$.

We have up to now considered a $2000 \mathrm{~km}$ thick conductive shell, but an infinite number of acceptable solutions exist which belong to the class of conductivity distributions having the same kernels $k_{n}(n=1,4)$. Limiting ourselves to the subclass of constant conductivity distributions, the curves shown in Figure 9 give the $\left(r_{\sigma}-r_{c}, \sigma\right)$ pairs equivalent, from the point of view of the present computation, to $\left(r_{\sigma}-r_{c}=2000 \mathrm{~km}\right.$, $\sigma=8 \mathrm{~S} \mathrm{~m}^{-1}$ ). Of course, the computations could be extended to any regular $\sigma(r)$ distribution.

\section{Discussion}

The present study is based on a simplistic two-shell model of the Earth, the outer insulating and the inner having a constant conductivity. It is known that the conductivity of the upper mantle is very low (< $1 \mathrm{~S} \mathrm{~m}^{-1}$ ) [Achache et al., 1981; Constable, 1993; Petersons and Constable, 1996], and it is likely that the 


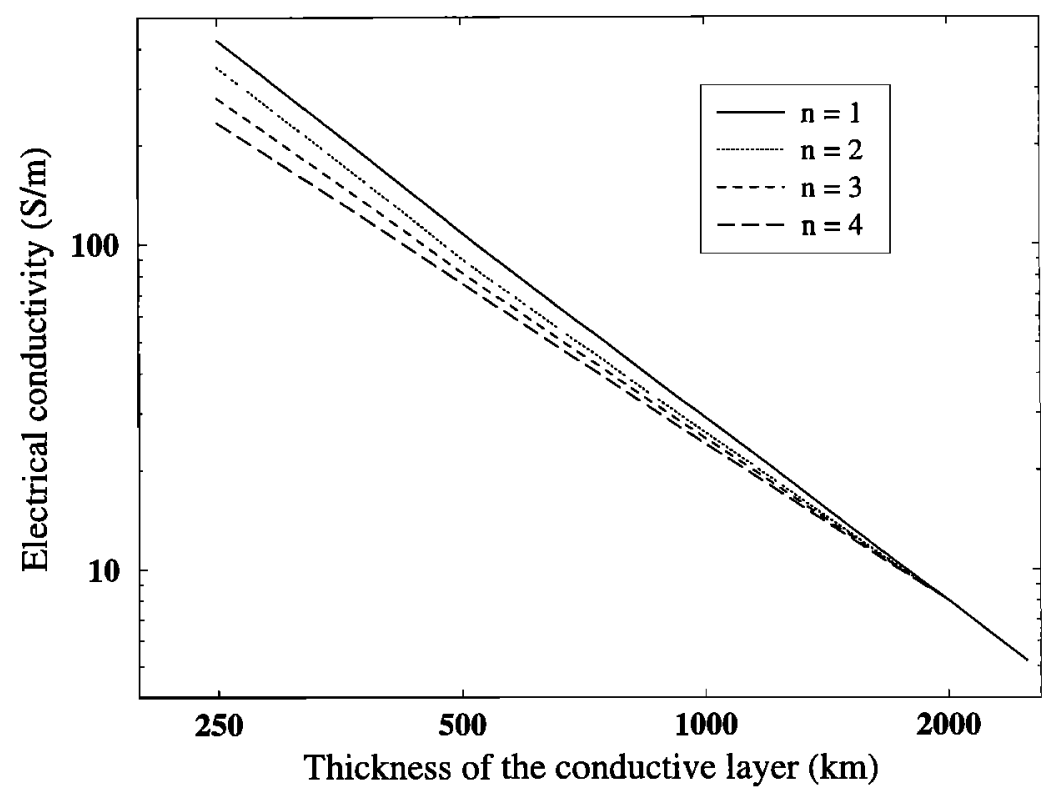

Figure 9. Relationship between the thickness $r_{\sigma}-r_{c}$ of the conductive layer and its conductivity $\sigma$ giving the same transfer fonctions $k_{n}(t)$ as those obtained with $r_{\sigma}-r_{c}=2000 \mathrm{~km}$ and $\sigma=$ $8 \mathrm{Sm}^{-1}$. For example, for $n=1$ the diffusive effects of a layer with $r_{\sigma}-r_{c}=2000 \mathrm{~km}$ and $\sigma=8 \mathrm{Sm}^{-1}$ and of a layer with $r_{\sigma}-r_{c}=500 \mathrm{~km}$ and $\sigma=108 \mathrm{Sm}^{-1}$ are identical.

conductivity distribution (as a function of depth) of the silicate lower mantle is regular [Shankland et al., 1993]. Our upper bound estimate bears in fact on the electromagnetic time constant $\tau$ of the mantle, translated in terms of the conductivity of an inner shell. Of course, most of the contribution to $\tau$ could be concentrated in a thin layer at the bottom of the mantle (reducing $\left(r_{\sigma}-r_{c}\right)^{2}$ and increasing $\left.\sigma\right)$. In order to establish the existence of such a layer, other kinds of evidence have to be found. Buffett et al. [1990], for example, proposed ohmic dissipation in a thin conducting layer at the base of the mantle as the source of a small residual out-ofphase component in the retrograde annual nutation of the Earth. A heterogeneous high-conductivity layer has also been invoked to explain the preferred geomagnetic virtual pole (VPG) paths during reversals [Laj et al., 1991; Runcorn, 1992]. However, some investigators find it difficult to explain the formation and existence of such a thin highly conductive layer at the base of the mantle [Pourier and Le Mouel, 1992; Le Mouel et al., 1997; Poirier et al., 1998; Katsura et al., 1998]. We are inclined to conclude that the conductivity of the lower mantle is basically that of solid silicates and oxides for which diamond anvil experiments [Poirier and Peyronneau, 1992; Shankland et al., 1993; Poirier et al., 1996] indicate a conductivity distribution which is weak and slowly increasing with depth; in general agreement with the estimates we obtain in the present study.

It should be emphasized that our study relies on the assumption that a jerk is a singularity at the CMB. Some assumptions are necessary in studies of the mantle filter for which we know the output but not the input.
The assumption that the jerk originated as a singularity introduced at the base of the mantle is the simplest possible.

Acknowledgments. We thank David Barraclough and Cathy Constable for comments. This study has been partly funded by the CNRS-INSU program "Intérieur de la Terre." This is IPGP contribution 1599.

\section{References}

Achache, J., V. Courtillot, J. Ducruix, and J.-L. Le Mouël, The late 1960's secular variation impulse: further constraints on deep mantle conductivity, Phys. Earth Planet. Inter., 23, 72-75, 1980.

Achache, J., J.-L. Le Mouël, and V. Courtillot, Long-period geomagnetic variations and mantle conductivity: An inversion using Bailey's method, Geophys. J. R. Astron. Soc., 65, 579-601, 1981.

Alexandrescu, M., D. Gibert, G. Hulot, J.-L. Le Mouël, and G. Saracco, Detection of geomagnetic jerks using wavelet analysis, J. Geophys. Res., 100, 12557-12572, 1995.

Alexandrescu, M., D. Gibert, G. Hulot, J.-L. Le Mouël, and G. Saracco, Worldwide wavelet analysis of geomagnetic jerks, J. Geophys. Res., 101, 21975-21994, 1996.

Backus, G.E., Application of mantle filter theory to the magnetic jerk of 1969, Geophys. J. R. Astron. Soc., 74, 713746,1983

Backus, G.E., R.L. Parker, and C. Constable, Foundations of Geomagnetism, 369 pp., Cambridge Univ. Press, New York, 1996.

Benton, E., and K. Whaler, Rapid diffusion of the poloidal geomagnetic field through the weakly conducting mantle: A perturbation solution, Geophys. J. R. Astron. Soc., 75, 77-100, 1983.

Buffett, B., T. Herring, P. Mathews, and I. Shapiro, Anomalous dissipation in the Earth's forced nutation: Inferences 
on electrical conductivity at the core-mantle boundary, Eos Trans. $A G U, 71,496,1990$.

Constable, S., Constraints on mantle electrical conductivity from field and laboratory measurements, J. Geomagn. Geoelectr., 45, 707-728, 1993.

Courtillot, V., and J.-L. Le Mouël, Geomagnetic secular variation impulses, Nature, 311, 709-716, 1984.

Courtillot, V., J. Ducruix, and J.-L. Le Mouël, Sur une accélération récente de la variation séculaire du champ magnétique terrestre, C. R. Acad. Sci., Ser. D, 287, 1095-1098, 1978

Courtillot, V., J.-L. Le Mouël, and J. Ducruix, On Backus' mantle filter theory and the 1969 geomagnetic impulse, Geophys. J. R. Astron. Soc., 78, 619-625, 1984.

De Michelis, P., L. Cafarella, and A. Meloni, Worldwide character of the 1991 jerk. Geophys. Res. Lett., 25, 377 $380,1998$.

Ducruix, J., V. Courtillot, and J.-L. Le Mouel, The late 1960 s secular varjation impulse, the 11 year magnetic variation and the electrical conductivity of the deep mantle, Geophys. J. R. Astron. Soc., 61, 73-94, 1980.

Gavoret, J., D. Gibert, M. Menvielle, and J.-L. Le Mouël, Long-term variations of the external and internal components of the Earth's magnetic field, J. Geophys. Res., 91, 4787-4796, 1986.

Golovkov, V.P., T.I. Zvereva, and A.O. Simonyan, Common feature and differences between "jerks" of 1947, 1958, and 1969, Geophys. Astrophys. Fluid Dyn., 49, 81-96, 1989.

Grossmann, A., Wavelet transform and edge detection, in Stochastic Processes in Physics and Engineering, edited by M. Hazewinkel, pp. 149-157, D. Reidel, Norwell, Mass., 1986.

Gubbins, D., Geomagnetic field analysis, II, Secular variation consistent with a perfectely conducting core, Geophys. J. R. Astron. Soc., 77, 753-766, 1984.

Gubbins, D., and L. Tomlinson, Secular variation from monthly means from Apia and Amberley magnetic observatories, Geophys. J. R. Astron. Soc., 86, 603-616, 1986.

Holschneider, M., Wavelets: An analysis tool, 423pp., Oxford Univ. Press, New York, 1995.

Hulot, G., J.-L. Le Mouël, and J. Wahr, Taking into account truncation problems and geomagnetic model accuracy in assessing computed flows at the core-mantle boundary, Geophys. J. Int., 108, 224-246, 1992.

Katsura T., K. Sato, and E. Ito, Electrical conductivity of silicate perovskite at lower-mantle conditions. Nature. 395, 493-495, 1998.

Kerridge, D.J., and D.R. Barraclough, Evidence for geomagnetic jerks from 1931 to 1971, Phys. Earth Planet. Inter. $39,228-236,1985$

Laj, C., A. Mazaud, R. Weeks, M. Fuller, and E. HerreroBervera, Geomagnetic reversal paths, Nature, 351, 447, 1991.

Le Huy, M., M. Alexandrescu, G. Hulot, and J.-L. Le Mouël, On the characteristics of successive geomagnetic jerks, Earth Planets Space, 50, 723-732, 1998.

Le Mouël, J.-L., L'induction dans le globe, in Traité de Géophysique Interne, vol. 2, edited by J. Coulomb and G. Jobert, pp. 129-158, Masson, Paris, Vol. 2, 1976.

Le Mouël, J.-L., G. Hulot, and J.-P. Poirier, Core-mantle interaction, in Earth's Deep Interior, edited by D.J. Crossley, pp. 197-221, Gordon and Breach, New York, 1997.

Macmillan, S., A geomagnetic jerk for the early 1990's, Earth Planet. Sci. Lett., 137, 189-192, 1996.
Malin, S., and B. Hodder, Was the 1970 geomagnetic jerk of internal or external origin?, Nature, 296, 726-728, 1982.

Malin, S., B. Hodder, and D. Barraclough, Geomagnetic secular variation: A jerk in 1970, Ebro Obs. Mem. Publ., 14, 239-256, 1983.

McLeod, M.G., Signals and noise in magnetic observatory annual means: Mantle conductivity and jerks, J. Geophys. Res., 97, 17261-17290, 1992.

McLeod, M.G., Magnetospheric and ionospheric signals in magnetic observatory monthly means: electrical conductivity of the deep mantle, J. Geophys. Res., 99, 1357713590, 1994.

Petersons, H.F., and S. Constable, Global mapping of the electrically conductive lower mantle, Geophys. Res. Lett. 23, 1461-1464, 1996.

Petiau, G., La Théorie des Fonctions de Bessel, 477 pp. Cent. Nat. de Rech. Sci., Paris, 1955.

Poirier, J.-P., and J.-L. Le Mouël, Does infiltration of the core into the lower mantle affect the observed geomagnetic field?, Phys. Earth Planet. Inter., 73, 29-37, 1992.

Poirier, J.-P., and J. Peyronneau, Experimental determination of the electrical conductivity of the material of the Earth's lower mantle, in High-Pressure Research: Applacation to Earth and Planetary Sciences, Geophys Monogr. Ser., vol. 67, edited by Y. Syono and M. Manghnani, pp. 77-87, AGU, Washington, D.C., 1992.

Poirier, J.-P., A. Goddat, and J. Peyronneau, Ferric iron dependence of the electrical conductivity of the Earth's lower mantle material, Philos. Trans. R. Soc. London, Ser. A, 354, 1361-1369, 1996.

Poirier, J.-P., V. Malavergne, and J.-L. Le Mouël, Is there a thin electrically conducting layer at the base of the mantle?, in The Core-Mantle Boundary Region, Geodyn. Ser., vol. 28, edited by M. Gurniset al., pp. 131-137, AGU, Washington, D.C., 1998.

Runcorn, S.K., The electrical conductivity of the Earth's mantle, Eos Trans. AGU, 36, 191-198, 1955.

Runcorn, S.K., Polar path in geomagnetic reversals, Nature, 356, 654-656, 1992.

Shankland, T.J., J. Peyronneau, and J.-P. Poirier, Electrical conductivity of the Earth's lower mantle, Nature, 366, 453-455, 1993.

Smylie, D.E., Magnetic diffusion in a spherically-symmetric conducting mantle, Geophys. J. R. Astron. Soc., 9, 169$184,1965$.

Stewart, D.N., and K.A. Whaler, Geomagnetic disturbance fields: An analysis of observatory monthly means, Geophys. J. Int., 108, 215-223, 1992.

Whaler, K.A., A new method for analysing geomagnetic impulses, Phys. Earth. Planet. Inter., 48, 221-240, 1987.

D. Gibert, and G. Saracco, Géosciences Rennes, Université de Rennes 1, Bât. 15 Campus de Beaulieu, 35042 Rennes cedex, France. (gibert@univ-rennes1.fr; ginet@univrennes1.fr)

G. Hulot, J.-L. Le Mouël, and M. Mandea Alexandrescu, Institut de Physique du Globe, B.P. 89, 4 Place Jussieu, 75252 Paris cedex 5, France. (ghulot@ipgp.jussieu.fr; lemouel@ipgp.jussieu.fr; mioara@ipgp.jussieu.fr)

(Received October 20, 1998; revised March 24, 1999; accepted April 2, 1999.) 